\title{
Duplication cyst of the cecum; a rare cause of intestinal obstruction in infants
}

\begin{abstract}
Although alimentary tract duplications are rare congenital anomalies which can occur anywhere along the gastrointestinal tract (GIT); cecal duplication is considered an extremely rare anomaly. We describe a 4-month-old female patient who were presented to our emergency department with a classical picture of acute intestinal obstruction in the form of abdominal distension and bilious vomiting warranting surgical exploration which revealed a duplication cyst at the cecalregion. Limited ileo-cecal resection along with the cyst was done with uneventful post-operative recovery with the diagnosis being confirmed by the pathological examination. Despite its rarity; cecal duplication should be put into consideration when exploring a neonate with intestinal obstruction.
\end{abstract}

Keywords alimentary tract duplication, cecal duplication, infant intestinal obstruction
Volume I0 Issue I - 2020

\author{
Ahmed Elrouby, Mostafa Zain, Ahmed \\ Hassan, Mansour Abdelkader, Ahmed \\ Aboelela \\ Pediatric Surgery Department, Faculty of Medicine,Alexandria \\ University, Egypt
}

\section{Correspondence: Ahmed Elrouby, Pediatric Surgery Department, Faculty of Medicine, Alexandria University, Egypt, Tel 0I22363I687,Email elroubypaedo@yahoo.com}

Received: November 27, 2019 | Published: January 10, 2020

\section{Introduction}

Although, it was said that Calder was the first to report similar lesions in 1733; the term (duplication of the alimentary tract) was introduced by Ladd in 1930s. ${ }^{1}$ Duplications of the GIT can affect any part of the alimentary tract from the oesophagus to the anus and always develop on the mesenteric border. Ileal and jejuna duplications are the commonest encountered duplications. On the other hand, cecal duplications are very rare subtype with an incidence of only $3 \%{ }^{2} \mathrm{We}$ report a case of 4- months old female patient who were presented to our department with a classical picture of intestinal obstruction. She had been urgently explored with a duplication cyst of the cecum being detected.

\section{Case Report}

A full-term four months old female with normal antenatal scans who was born by spontaneous vaginal delivery without any reported perinatal morbidities was presented to our department with a classical picture of intestinal obstruction in the form of vomiting, abdominal distention and non-passage of stool.

Her parents told us that the condition started with non-bilious vomiting since 2 days which progressed to bilious vomiting with increased frequency along with progressive abdominal distension and obstipation. They claimed that the baby was previously well without any history of intermittent colicky abdominal pain, passage of bloody stool nor any previous similar attacks of bilious vomiting.

Systemic examination revealed that the patient was dehydrated, lethargic and tachycardic. Urgent resuscitation with I.V. normal Saline $(10 \mathrm{mg} / \mathrm{kg})$ was then started with marvellous improvement of her general condition. The nasogastric tube drained $100 \mathrm{ml}$ of bilious suction. Chest examination showed equal bilateral air entry with no adventitious sounds. There was no obvious spinal nor other associated congenital anomalies.

Local abdominal examination revealed marked abdominal distension with dilated veins and visible bowel loops without any sign of peritonitis. Normal groin examination with no obvious hernias with empty rectum on digital rectal examination. Normally located anus with normally looking perineum were also noticed.
Laboratory investigations revealed average values except for slight hypokalaemia which was corrected pre-operatively.

Standing abdominal X-ray showed marked abdominal distension with multiple air-fluid levels suggesting distal ileal obstruction, with no obvious masses or free air under the diaphragm. (Figure 1).

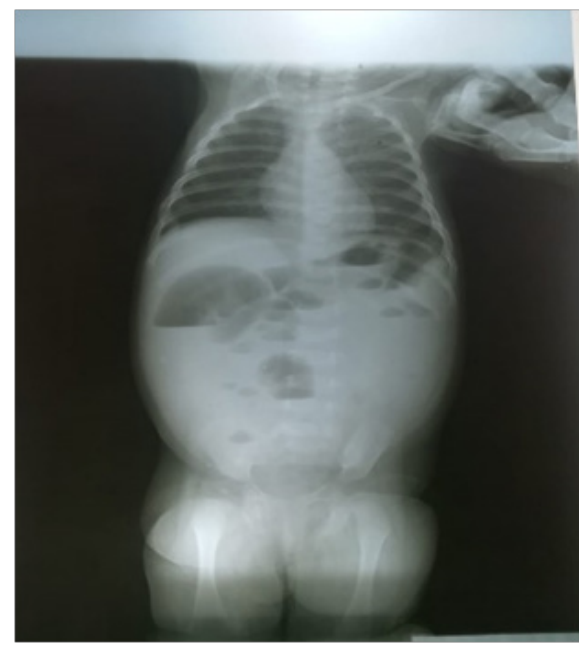

Figure I PXR abdomen standing showing multiple air fluid levels.

Urgent US abdomen revealed a well-defined fluid filled thick walled cystic structure at the right hypochondrial region measuring about $3 \mathrm{~cm} \times 2.5 \mathrm{~cm}$ with marked proximal small bowel dilation.

Owing to the marked abdominal distention; laparoscopy was not feasible and the decision was to proceed with lower midline exploratory laparotomy. Exploration revealed a cystic lesion on the mesenteric side of the cecum. (Figure 2).

The small intestinal loops were distended with collapsed large gut. There was complete intestinal obstruction at the level of the cyst as shown by failure of the passage of the intestinal contents distal to the cyst using manual milking. The posterior wall of the cyst shared a common wall with the cecum. Resection of the ileo-cecalregion with the cystic mass was done with ileo-ascending end to end anastomosis using a single layer of interrupted Vicryl 4/0 sutures. 


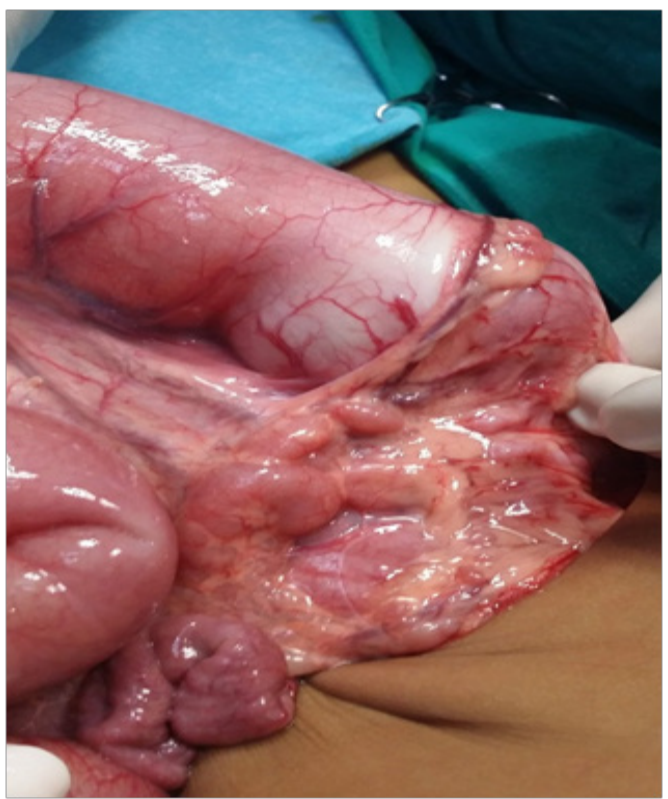

Figure 2 Intra-operative view of the cecal cyst.

The $8 \mathrm{~cm}$ excised segment was sent for the histopathological examination which report the presence of a cystic lesion at the mesenteric border of the cecum measuring $3 \mathrm{~cm} \times 2.5 \mathrm{~cm}$. (Figure $3 \mathrm{~A}$, B).
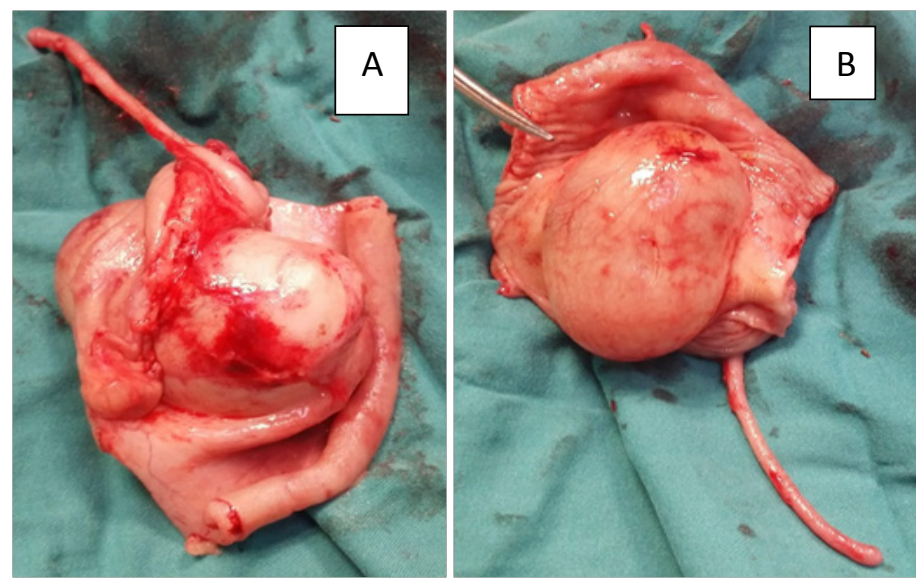

Figure 3(A-B) The resected ileo-cecal specimen showing the cyst on the mesenteric border.

Microscopic examination of the noted cyst showed the presence of all the layers of large intestine with diffuse chronic inflammatory infiltrate of the mucosa and submucosa in association with prominent lymphoid follicles in the submucosa. The muscularispropria was thickened with congested and dilated blood vessels. There was no evidence of ectopic or abnormal tissue.

The Patient passed an uneventful recovery, started oral feeding on the $2^{\text {nd }}$ post-operative day and was discharged home on $4^{\text {th }}$ postoperative day. The child was followed up for 6 months and she was apparently normal.

\section{Discussion}

In 1937 WE Ladd introduced the term of alimentary tract duplications to unify the nomenclature of this wide spectrum of anomalies that involve the mesenteric side of the associated alimentary tract sharing its common blood supply. The most common site of duplication is the Ileum. ${ }^{3}$

Colonic duplication cysts are very rare $(13 \%$ of duplications of the alimentary tract).Cecal duplication cysts are even rarer as only 9 cases were reported in the English literature. $80 \%$ of these cases present in the first 2 years of life, but it has also been reported in adults. ${ }^{4}$

More than $60 \%$ of cases who have alimentary duplication present in the first 2 years of life. Some of these cases are associated with other congenital anomalies like vertebral anomalies. ${ }^{5}$ Split notochord syndrome is considered the most satisfactory of the numerous theories which explain the origin of alimentary tract duplication. This theory relates the origin of this anomaly to maldevelopment of the neuro-enteric canal. Other theories include partial twinning theory, canalization defect theory and environmental factors theory. ${ }^{1}$

Symptoms vary according to the nature of the cyst, size, shape, and site. etc. It may present with abdominal pain, distension, vomiting, upper or lower GI bleeding or intestinal obstruction. The mechanism of obstruction in cecal duplication depends upon the amount of mucous in its lumen. Fully loaded cecal duplication can obstruct the lumen of the normal cecum and may result in acute intestinal obstruction as in our case. ${ }^{1}$

The clinical diagnosis of this anomaly is very difficult and it is usually discovered incidentally during operative exploration of another cause. Laboratory and radiological investigations in this situation are also non-specific.

Pathological evaluation of the enteric cysts is the mainstay of diagnosis. Gross and microscopic appearance showing mucosa, submucosa, muscularis, and serosa are the typical features. Good sectioning of the cyst wall with the attached bowel helps in ruling out the malignant changes. ${ }^{3}$

Various surgical procedures have been employed to deal with such lesions. Most commonly used is the partial colectomy with end-to-end anastomosis as in our case. ${ }^{5}$ Other techniques such as enucleation, marsupialization, or evacuation of the cyst can also be used in selected cases. $^{1}$

\section{Conclusion}

Despite its rarity; cecal duplication should be put into consideration when exploring a neonate with intestinal obstruction.

\section{Acknowledgement \& Funding}

None.

\section{Conflict of interest}

The following authors have no Conflicts of interest.

\section{References}

1. Ijaz L, Husnain M, Malik SI, et al. Cecal duplication cyst presenting as acute intestinal obstruction in an infant. APSP J Case Rep. 2011;2(1):11. 
2. Mayer JP. Alimentary tract duplications in newborns and children Diagnostic aspects and the role of laparoscopic treatment. World $J$ Gastroenterol. 2014;20(39):14263.

3. Yisau A, Abdulraseed N, Kashim I, et al. Gastrointestinal duplications: Experience in seven children and a review of the literature. Saudi J Gastroenterol. 2010;16(2):105.
4. Singh VR, Shah P, Roplekar P, et al. Cecal duplication cyst: A rare case report with review of literature. Int J Heal Allied Sci. 2016;5(2):115.

5. Pati A, Mohanty HK, Subudhi PC, et al. Duplication cyst of the cecum: A case report. Indian J Surg. 2010;22(3):271-272. 